\title{
Electrohydrodynamic instability of a rotating couple stress dielectric fluid layer
}

\author{
I.S. Shivakumara ${ }^{\mathrm{a}}$, M. Akkanagamma ${ }^{\mathrm{a}}$, Chiu-On Ng ${ }^{\mathrm{b}, *}$ \\ ${ }^{a}$ UGC - CAS in Fluid Mechanics, Department of Mathematics, Bangalore University, \\ Bangalore-560 001, India. \\ ${ }^{b}$ Department of Mechanical Engineering, The University of Hong Kong, Pokfulam Road, \\ Hong Kong
}

\begin{abstract}
A linear stability analysis is performed to study the effect of a vertical alternating current (AC) electric field on the criterion for the onset of convection in a horizontal couple stress dielectric fluid layer heated uniformly either from below or from above. The lower and upper isothermal boundaries of the fluid layer are considered to be either rigid or free. The principle of exchange of stability is shown to be valid irrespective of the nature of velocity boundary conditions. The resulting eigenvalue problem is solved exactly for free-free boundaries and numerically using the Galerkin method for rigid-rigid boundaries. The stability characteristics of the system have been analyzed when the electric and buoyancy forces are acting together as well as in isolation and their coupling effects are highlighted in the presence of couple stresses. It is noted that there is a qualitative agreement between the results of free-free and rigid-rigid boundaries. The effect of increasing AC electric Rayleigh number is to increase the transfer of heat more effectively and hence to hasten the onset of convection. To the contrary, the effect of increasing couple stress parameter is to delay the onset of electrothermal convection. Moreover, the influence of couple stress on the stability characteristics of the system is found to be more pronounced for the case with rigid boundaries compared to the free boundaries case.
\end{abstract}

Key words: dielectric fluid; electrothermal convection; couple stress fluid

\footnotetext{
*Corresponding author:

Email addresses: shivakumarais@gmail.com (I.S. Shivakumara), cong@hku.hk (C.O. Ng)
} 


\section{Nomenclature}

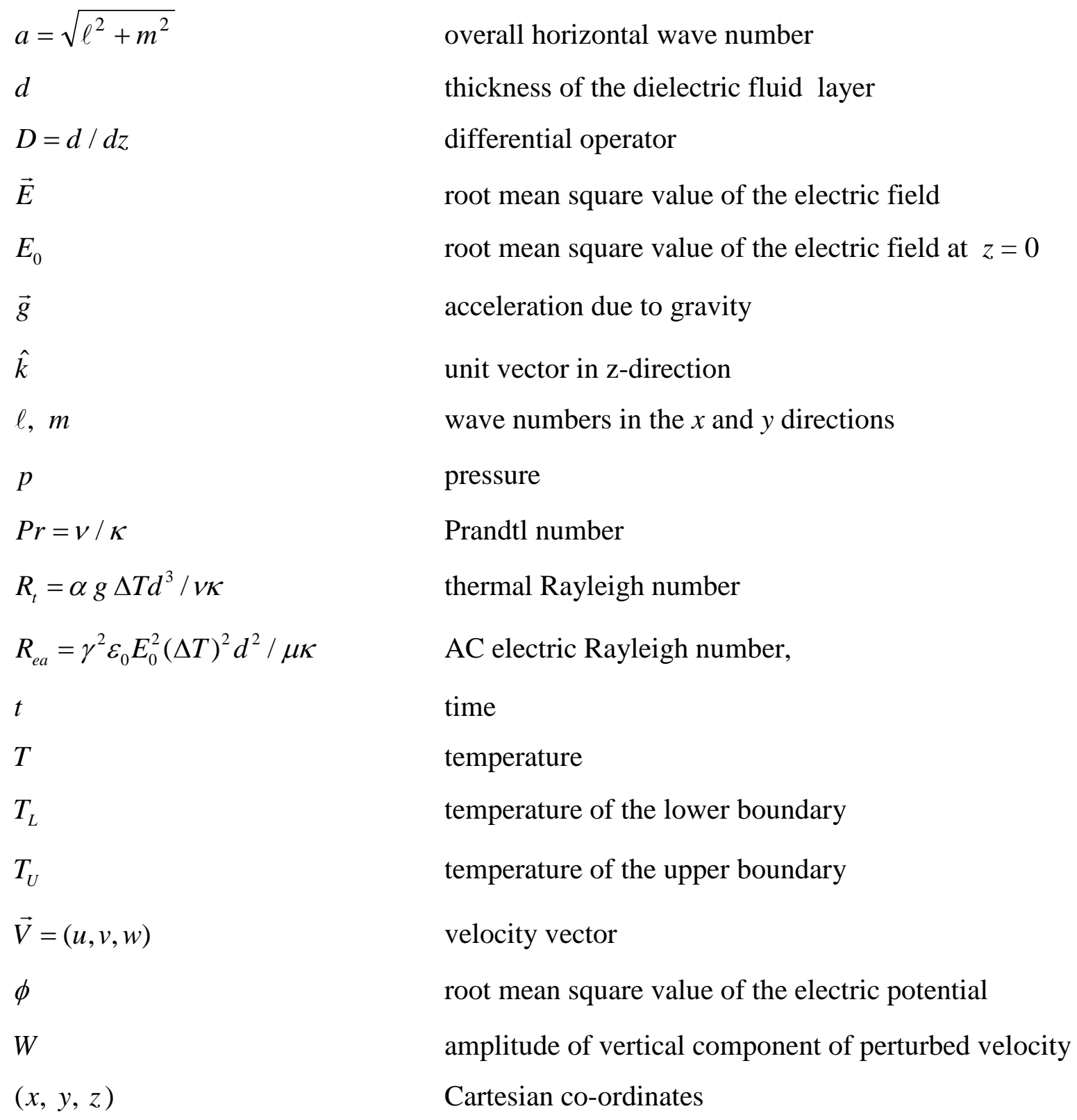

overall horizontal wave number

thickness of the dielectric fluid layer

differential operator

root mean square value of the electric field

root mean square value of the electric field at $z=0$

acceleration due to gravity

unit vector in $\mathrm{z}$-direction

wave numbers in the $x$ and $y$ directions

pressure

Prandtl number

thermal Rayleigh number

AC electric Rayleigh number,

time

temperature

temperature of the lower boundary

temperature of the upper boundary

velocity vector

root mean square value of the electric potential

amplitude of vertical component of perturbed velocity

Cartesian co-ordinates

\section{Greek symbols}

$\alpha$

$\gamma$

$\nabla_{h}^{2}=\partial^{2} / \partial x^{2}+\partial^{2} / \partial y^{2}$

$\nabla^{2}=\nabla_{h}^{2}+\partial^{2} / \partial z^{2}$

$\varepsilon$

$\varepsilon_{0}$

K thermal expansion coefficient

thermal expansion coefficient of dielectric constant

horizontal Laplacian operator

Laplacian operator

dielectric constant

reference dielectric constant at $T_{L}$

thermal diffusivity of the fluid 


$\begin{array}{ll}\sigma & \text { electrical conductivity of the fluid } \\ \mu & \text { fluid viscosity } \\ \mu_{c} & \text { couple stress viscosity } \\ \omega & \text { growth rate } \\ \rho & \text { fluid density } \\ \rho_{e} & \text { free charge density } \\ \rho_{0} & \text { reference density at } T_{L} \\ \Phi & \text { amplitude of perturbed } \phi \\ \Lambda_{c}=\mu_{c} / \mu d^{2} & \text { couple stress parameter } \\ \Theta & \text { amplitude of perturbed temperature }\end{array}$

\section{Introduction}

Thermal convective instability in a horizontal viscous fluid layer heated from below is one of the classical problems and it is considered to be a simple model system for a wide variety of geophysical, astrophysical and industrial flows. However, many convective instability problems of practical importance involve electrically conducting fluids. In such cases, the effect of external fields like magnetic and electric fields become important. In particular, the magnetic field effects become dominant if the fluid is highly electrically conducting. To the contrary, if the fluid is dielectric with low electrical conductivity then the electric forces play a major role in driving the motion due to the effect of specific electroconvective instability mechanisms. The interplay between electric field and fluid motion has led to many complex and interesting instability phenomena in microchannels. It has been realized that utilizing electric force to manipulate fluids is the most efficient method in the achievement of a variety of purposes and functions in the applications of microfluidic devices. A brief discussion on the applications of electrohydrodynamic (EHD) instability has been recently presented by Lin (2009).

In view of a possible control of convection in liquid dielectrics and a control of heat transfer in high-voltage devices, the study on convective instability in a horizontal dielectric fluid layer subject to a vertical temperature gradient and a vertical AC or DC electric field has constituted a pole of attraction to researchers. In such situations, an applied temperature gradient produces non-uniformities in the electrical conductivity and/or the dielectric 
permittivity. The variation of electrical conductivity of the fluid with temperature produces free charges in the bulk of the fluid. These free charges interacting with applied or induced electric field produce a force that eventually causes fluid motion. On the other hand, when there is variation in dielectric permittivity and the electric field is intense then the polarization force which is induced by the non-uniformity of the dielectric constant causes fluid motion. In either case, convection can occur in a dielectric fluid layer even if the temperature gradient is stabilizing and such an instability produced by an electric field is called electroconvection, which is analogous to Rayleigh-Benard convection. In addition, if the applied temperature gradient is also destabilizing then such an instability problem is called electrothermal convection.

Copious literature is available on the problem of the onset of convective instability in a horizontal dielectric fluid layer subject to a vertical temperature gradient and a vertical AC or DC electric field (Turnbull, 1968; Roberts, 1969; Takashima \& Aldridge, 1976; Bradley, 1978; Jones, 1978; Maekawa, Abe \& Tanasawa, 1992; Pontiga \& Castellanos, 1994; Saville, 1997; Smorodin, 2001; Straughan, 2004). The combined effects of DC electric field and volumetric heat source on the onset of convection in a dielectric fluid layer heated from below is investigated by Shivakumara, Nagashree, and Hemalatha (2007), while the influences of a vertical AC electric field as well as internal heat generation on the onset of electrothermoconvection in a horizontal dielectric fluid layer is analyzed by Shivakumara, Rudraiah, and Hemalatha (2009). A more detailed analysis on EHD instability in a horizontal fluid layer with electrical conductivity gradient subject to a weak shear flow is presented by Chang, Ruo, and Chen (2009). The electrohydrodynamic instability of a horizontal rotating fluid layer with a vertical electrical conductivity gradient has been considered by Ruo, Chang, and Chen (2010). Recently, Shivakumara, Lee, Vajravelu and Akkanagamma (2012) have studied the effect of velocity and temperature boundary conditions on electrothermal convection in a rotating dielectric fluid layer.

Although EHD instability has been extensively investigated in a dielectric Newtonian fluid layer, relatively little attention is devoted to the problem concerning non-Newtonian fluids despite their occurrence and importance in many practical problems. There exist various types of non-Newtonian fluids, and obviously they do not lend themselves to a unified treatment like Newtonian ones. A few studies have addressed the effect of vertical AC electric field on the onset of convection for one such type of non-Newtonian fluid called 
viscoelastic fluid. Takashima and Ghosh (1979) were the first to study the simultaneous action of a vertical AC electric field and a vertical temperature gradient on the onset of instability in a horizontal layer of viscoelastic dielectric fluid. Othman (2001) has studied the onset of convection in a viscoelastic dielectric fluid layer heated from below. Othman and Sweilam (2002) have examined the effect of vertical AC electric field on the stability of natural convection, which occurs in a dielectric viscoelastic fluid (Walters' liquid $B^{\prime}$ ) between two parallel horizontal plates with internal heat generation. Othman (2003) has considered the effect of thermal relaxation time on EHD instability in a viscoelastic dielectric fluid layer heated from below. The effect of rotation on EHD instability in a layer of viscoelastic dielectric fluid heated from below has been investigated by Othman (2004).

Nonetheless, consideration of non-Newtonian fluids with suspended particles is desirable as these fluids deform and produce a spin field due to the microrotation of suspended particles forming a micropolar fluid. The theory of micropolar fluids was developed by Eringen (1966) which takes care of local effects arising from the microstructure and as well as the intrinsic motions of microfluidics. The spin field, due to microrotation of freely suspended particles, sets up an anti-symmetric stress known as couple stress and thus forming couple stress fluid. The couple-stress fluid has distinct features, such as polar effects and whose microstructure is mechanically significant. Besides, couple stresses are found to appear in noticeable magnitude in fluids with large molecules. The constitutive equations for couple stress fluids are given by Stokes (1966). The theory proposed by Stokes is the simplest one for micro-fluids, which allows polar effects such as the presence of couple stress, body couple and non-symmetric tensors. These fluids have applications in a number of processes that occur in industry, such as the extrusion of polymer fluids, solidification of liquid crystals, cooling of metallic plate in a bath, exotic lubrication and colloidal and suspension solutions. The onset of convection in a couple stress fluid layer has also attracted researchers in the recent past (Malashetty, Gaikwad \& Swamy 2006; Sunil, 2011 and references therein). The effect of magnetic field and rotation on convective instability in a layer of couple stress fluid heated from below has been considered by Sharma and Sharma (2004).

In this paper attention is focused on the study of EHD instability in a layer of couple stress dielectric fluid in the presence of a vertical AC electric field. Interest in such a problem stems from the studies on buoyancy driven convective instability in a layer of couple stress fluid, in which the presence of couple stresses shows a significant effect on the stability 
characteristics of the system. The problem is discussed for two types of velocity boundary conditions namely, (i) both boundaries free (free-free), and (ii) both boundaries rigid (rigidrigid) with prescribed different temperatures at the boundaries. The eigenvalue problem is solved exactly for free-free boundaries, while numerically using the Galerkin method for rigid-rigid boundaries. The coupling effects of electrical body force, buoyancy force and the couple stresses on the onset of electrothermal convection are explored in detail.

\section{Formulation of the problem}

We consider a horizontal layer of thickness $d$ of an incompressible dielectric couple stress fluid in the absence of body couple with a uniform vertical AC electric field applied across the layer. A Cartesian coordinate system $(x, y, z)$ is chosen with the origin at the bottom of the fluid layer and the z- axis normal to the fluid layer in the gravitational field. The lower surface at $z=0$ and the upper surface at $z=d$ are maintained at temperatures $T_{L}$ and $T_{U}$, respectively. Furthermore, the lower surface is grounded and the upper surface is kept at an alternating $(60 \mathrm{~Hz})$ potential whose root- mean- square value is $f_{1}$.

The relevant basic equations under the Oberbeck-Boussinesq approximation are:

$$
\begin{aligned}
& \nabla \cdot \vec{V}=0 \\
& \rho_{0}\left[\frac{\partial \vec{V}}{\partial t}+(\vec{V} \cdot \nabla) \vec{V}\right]=-\nabla P+\rho \vec{g}+\mu \nabla^{2} \vec{V}-\mu_{c} \nabla^{4} \vec{V}-\frac{1}{2}(\vec{E} \cdot \vec{E}) \nabla \varepsilon \\
& \frac{\partial T}{\partial t}+(\vec{V} \cdot \nabla) T=\kappa \nabla^{2} T \\
& \rho=\rho_{0}\left\{1-\alpha\left(T-T_{L}\right)\right\}
\end{aligned}
$$

where $\vec{V}=(u, v, w)$ is the velocity vector, $T$ is the temperature, $\vec{E}$ is the root-mean-square value of the electric field, $P=p-\frac{\rho}{2} \frac{\partial \varepsilon}{\partial \rho}(\vec{E} \cdot \vec{E})$ is the modified pressure, $\varepsilon$ is the dielectric constant, $\rho$ is the fluid density, $\kappa$ is the thermal diffusivity, $\mu$ is the fluid viscosity, $\mu_{c}$ is the material constant responsible for the couple-stress property known as the couple stress viscosity, $\vec{g}$ is the acceleration due to gravity, $\alpha$ is the thermal expansion coefficient, $\rho_{0}$ is the density at reference temperature $T=T_{L}$. The Coulomb force term $\rho_{e} \vec{E}$, where $\rho_{e}$ is the free charge density, is of negligible order compared with the dielectrophoretic force term for most dielectric fluids in a 60-Hz AC electric field (Takashima and Ghosh, 1979). Therefore, 
the Coulomb force term has been neglected in Eq. (2) and only the dielectrophoretic force term is retained i.e., the last term in Eq. (2). It is seen that the dielectrophoretic force term depends on $(\vec{E} \cdot \vec{E})$ rather than $\vec{E}$. Since the variation of $\vec{E}$ is very rapid, the root-meansquare value of $\vec{E}$ is used as the effective value in determining fluid motions.

Since there is no free charge, the relevant Maxwell equations are

$$
\nabla \times \vec{E}=0, \nabla \cdot(\varepsilon \vec{E})=0 .
$$

In view of Eq.(5a), $E$ can be expressed as

$$
\text { É - ? } f
$$

where $f$ is the root mean square value of the electric potential. The dielectric constant is assumed to be a linear function of temperature in the form

$$
\varepsilon=\varepsilon_{0}\left[1-\gamma\left(T-T_{L}\right)\right]
$$

where $\gamma(>0)$ is the thermal expansion coefficient of dielectric constant and is assumed to be small.

\subsection{Basic state}

The basic state is quiescent and is given by

$$
\begin{aligned}
& \vec{V}=\vec{V}_{b}=0, P=P_{b}(z), T=T_{b}(z)=T_{L}-\mathrm{D} T z / d \\
& \varepsilon=\varepsilon_{b}(z)=\varepsilon_{0}[1+\gamma \Delta T z / d] \\
& \stackrel{r}{E}=\stackrel{r}{E_{b}}(z)=\frac{E_{0}}{(1+g \mathrm{D} T z / d)} \hat{k}
\end{aligned}
$$

where $\mathrm{DT}=T_{L}-T_{U}$ and the subscript $b$ denotes the basic state. The pressure distribution is of no consequence as we are going to eliminate the same. We note that

$$
f_{b}(z)=-\frac{E_{0} d}{g \mathrm{D} T} \log (1+g \mathrm{DTz} / d)
$$

where

$$
E_{0}=-\frac{f_{1} g \mathrm{D} T / d}{\log (1+g \mathrm{D} T)}
$$

is the root mean square value of the electric field at $z=0$.

\subsection{Perturbed state}

To study the stability of the basic state, we superimpose infinitesimally small perturbations on the basic state in the form 


$$
\vec{V}=\vec{V}^{\prime}, P=P_{b}+P^{\prime}, \vec{E}=\vec{E}_{b}+\vec{E}^{\prime}, T=T_{b}+T^{\prime}, \rho=\rho_{b}+\rho^{\prime}, \varepsilon=\varepsilon_{b}+\varepsilon^{\prime}
$$

where ${ }^{\prime}, \mathcal{P},{ }^{\prime}, T, r$ and $e$ are the perturbed quantities over their equilibrium counterparts. Substituting Eq.(10) into Eqs. (1) - (7), linearizing the equations, eliminating the pressure from the momentum equation by operating curl twice and retaining the vertical component and non-dimensionalizing the resulting equations by scaling $(x, y, z)$ by $d$, $\mathrm{t}$ by $d^{2} / \kappa, \vec{V}^{\prime}$ by $\kappa / d, T^{\prime}$ by $\Delta T$ and $f \Phi$ by $\gamma E_{0} \Delta T d$, we obtain the linear stability equations ( after neglecting the primes for simplicity) in the form

$$
\begin{aligned}
& \left(\frac{1}{\operatorname{Pr}} \frac{\partial}{\partial t}-\nabla^{2}+\Lambda_{c} \nabla^{4}\right) \nabla^{2} w=R_{t} \nabla_{h}^{2} T+R_{e a} \nabla_{h}^{2}\left(T-\frac{\partial \phi}{\partial z}\right) \\
& \left(\frac{\partial}{\partial t}-\nabla^{2}\right) T=w \\
& \nabla^{2} \phi=\frac{\partial T}{\partial z}
\end{aligned}
$$

where $R_{t}=\alpha g \Delta T d^{3} / v \kappa$ is the thermal Rayleigh number, $R_{e a}=\gamma^{2} \varepsilon_{0} E_{0}^{2}(\Delta T)^{2} d^{2} / \mu \kappa$ is the AC electric Rayleigh number, $\Lambda_{c}=\mu_{c} / \mu d^{2}$ is the couple stress parameter and $\operatorname{Pr}=v / \kappa$ is the Prandtl number.

The isothermal boundaries of the fluid layer are considered to be either stress-free or rigid with vanishing couple stress. The boundary conditions are:

$$
w=\frac{\partial^{2} w}{\partial z^{2}}=\frac{\partial^{4} w}{\partial z^{4}}=\frac{\partial \phi}{\partial z}=0, T=0
$$

on the stress-free boundary and

$$
w=\frac{\partial w}{\partial z}=\frac{\partial^{3} w}{\partial z^{3}}=\phi=0, \quad T=0
$$

on the rigid boundary.

Equations (11)-(13) are simplified in the usual manner by decomposing the solution in terms of normal modes, so that

$$
(w, T, \phi)=(W, \Theta, \Phi)(z) \exp (i \ell x+i m y+\omega t)
$$

where $\omega\left(=\omega_{r}+i \omega_{i}\right)$ is the growth rate, $\ell$ and $m$ are the horizontal wave numbers in the $x$ and $y$ directions respectively. Substituting Eq. (16) into Eqs. (11) - (13), we obtain the following stability equations: 


$$
\begin{aligned}
& \left\{\frac{\omega}{P r}-\left(D^{2}-a^{2}\right)+\Lambda_{c}\left(D^{2}-a^{2}\right)^{2}\right\}\left(D^{2}-a^{2}\right) W=-\left(R_{t}+R_{e a}\right) a^{2} \Theta+R_{e a} a^{2} D \Phi \\
& \left\{\omega-\left(D^{2}-a^{2}\right)\right\} \Theta=W \\
& \left(D^{2}-a^{2}\right) \Phi=D \Theta
\end{aligned}
$$

where $D=d / d z$ and $a=\sqrt{\ell^{2}+m^{2}}$ is the overall horizontal wave number.

On using Eq. (16) in the boundary conditions (14) and (15), we get respectively

$$
W=D^{2} W=D^{4} W=D \Phi=0, \Theta=0
$$

and

$$
W=D W=D^{3} W=\Phi=0, \Theta=0
$$

\section{Validity of principle of exchange of stability}

To establish this, we first eliminate $\Phi$ from Eq. (17) by operating $\left(D^{2}-a^{2}\right)$ throughout and then use Eq. (19) to obtain

$$
\left[\frac{\omega}{\operatorname{Pr}}-\left(D^{2}-a^{2}\right)+\Lambda_{c}\left(D^{2}-a^{2}\right)^{2}\right]\left(D^{2}-a^{2}\right)^{2} W=-R_{t} a^{2} D^{2} \Theta+\left(R_{t}+R_{e a}\right) a^{4} \Theta .
$$

Also, we have

$$
\left[\omega-\left(D^{2}-a^{2}\right)\right] \Theta=W
$$

Multiplying Eq. (22) by $W^{*}$ (the complex conjugate of $W$ ) and integrating from $z=0$ to 1 , we obtain

$$
\begin{aligned}
<W^{*}\left[\frac{\omega}{\operatorname{Pr}}-\left(D^{2}-a^{2}\right)\right. & \left.+\Lambda_{c}\left(D^{2}-a^{2}\right)^{2}\right]\left(D^{2}-a^{2}\right)^{2} W> \\
& =-R_{t} a^{2}<W^{*} D^{2} \Theta>+\left(R_{t}+R_{e a}\right) a^{4}<W^{*} \Theta>
\end{aligned}
$$

where, $\langle\cdots\rangle=\int_{0}^{1}(\cdots) d z$. On using the velocity boundary conditions and repeatedly applying integration by parts and simplifying, the LHS of Eq.(24) can be written as

$$
\begin{aligned}
\frac{\omega}{\operatorname{Pr}}<\left|D^{2} W\right|^{2} & +2 a^{2}|D W|^{2}+a^{4}|W|^{2}>+<\Lambda_{\mathrm{c}}\left|D^{4} W\right|^{2}+\left(1+4 a^{2} \Lambda_{\mathrm{c}}\right)\left|D^{3} W\right|^{2}> \\
& +<3 a^{2}\left(1+2 a^{2} \Lambda_{\mathrm{c}}\right)\left|D^{2} W\right|^{2}+a^{4}\left(3+4 a^{2} \Lambda_{\mathrm{c}}\right)|D W|^{2}+a^{6}\left(1+a^{2} \Lambda_{\mathrm{c}}\right)|W|^{2}>.
\end{aligned}
$$

From Eq. (23), we have 


$$
\left[\omega^{*}-\left(D^{2}-a^{2}\right)\right] \Theta^{*}=W^{*}
$$

The right hand side of Eq. (24) on using Eq. (26) becomes

$$
\begin{aligned}
R_{t} a^{2}\left[\omega^{*}\right. & \left.<|D \Theta|^{2}>+<\left|D^{2} \Theta\right|^{2}+a^{2}|D \Theta|^{2}>\right] \\
& +\left(R_{t}+R_{e a}\right) a^{4}\left[\omega^{*}<|\Theta|^{2}>+<|D \Theta|^{2}+a^{2}|\Theta|^{2}>\right] .
\end{aligned}
$$

Use of Eq.(25) and Eq.(27) in Eq.(24) gives

$$
\begin{aligned}
& \frac{\omega}{P r}<\left|D^{2} W\right|^{2}+2 a^{2}|D W|^{2}+a^{4}|W|^{2}>+<\Lambda_{\mathrm{c}}\left|D^{4} W\right|^{2}+\left(1+4 a^{2} \Lambda_{\mathrm{c}}\right)\left|D^{3} W\right|^{2}> \\
&+<3 a^{2}\left(1+2 a^{2} \Lambda_{\mathrm{c}}\right)\left|D^{2} W\right|^{2}+a^{4}\left(3+4 a^{2} \Lambda_{\mathrm{c}}\right)|D W|^{2}+a^{6}\left(1+a^{2} \Lambda_{\mathrm{c}}\right)|W|^{2}> \\
&=R_{t} a^{2}<\left|D^{2} \Theta\right|^{2}+a^{2}|D \Theta|^{2}>+\left(R_{t}+R_{e a}\right) a^{4}<|D \Theta|^{2}+a^{2}|\Theta|^{2}> \\
&+\omega^{*}\left[R_{t} a^{2}<|D \Theta|^{2}>+\left(R_{t}+R_{e a}\right) a^{4}<|\Theta|^{2}>\right]
\end{aligned}
$$

The real and imaginary parts of the above equation are equated separately. The real part, in effect, determines whether the system returns to the equilibrium position or not depending on the sign of the real part of $\omega$. The imaginary part gives the information about the possibility of occurring oscillatory instability. Equating the imaginary parts of Eq. (28), we obtain

$$
\begin{aligned}
\frac{\omega_{i}}{P r}<\left|D^{2} W\right|^{2} & +2 a^{2}|D W|^{2}+a^{4}|W|^{2}> \\
& =-\omega_{i}\left[R_{t} a^{2}<|D \Theta|^{2}>+\left(R_{t}+R_{e a}\right) a^{4}<|\Theta|^{2}>\right] .
\end{aligned}
$$

Thus oscillatory convection occurs only if,

$$
\frac{1}{P r}<\left|D^{2} W\right|^{2}+2 a^{2}|D W|^{2}+a^{4}|W|^{2}>+R_{t} a^{2}<|D \Theta|^{2}>+\left(R_{t}+R_{e a}\right) a^{4}<|\Theta|^{2}>=0 .
$$

But the above condition will never be satisfied as $R_{e a} \geq 0$ and the left hand side of Eq.(30) is positive definite. This establishes that oscillatory instability cannot occur in a layer of couple stress dielectric fluid heated uniformly from below under the influence of a vertical AC electric field. Therefore, we restrict ourselves to stationary convection in our subsequent analyses and take $\omega=0$ in Eqs. (17) and (18) to arrive at the following stability equations:

$$
\begin{aligned}
& \left\{\Lambda_{c}\left(D^{2}-a^{2}\right)^{2}-\left(D^{2}-a^{2}\right)\right\}\left(D^{2}-a^{2}\right) W=-\left(R_{t}+R_{e a}\right) a^{2} \Theta+R_{e a} a^{2} D \Phi \\
& \left(D^{2}-a^{2}\right) \Theta=-W \\
& \left(D^{2}-a^{2}\right) \Phi=D \Theta .
\end{aligned}
$$




\section{Solution}

Equations (31)-(33) together with the chosen boundary conditions constitute an eigenvalue problem for $R_{e a}$ or $R_{t}$ as functions of the remaining parameters. The instability characteristics are explored for two types of velocity boundary conditions namely, both boundaries free (free-free) and both boundaries rigid (rigid-rigid).

\subsection{Both boundaries free}

Although the free boundaries are admittedly artificial, it is of importance because the solution to the eigenvalue problem can be obtained exactly and thereby the essential features of the problem can be disclosed. Eliminating $\Theta$ and $\Phi$ between Eqs. (31)-(33), we obtain an equation in $W$ in the form

$$
\left\{\Lambda_{c}\left(D^{2}-a^{2}\right)^{2}-\left(D^{2}-a^{2}\right)\right\}\left(D^{2}-a^{2}\right)^{3} W=R_{t} a^{2}\left(D^{2}-a^{2}\right) W-R_{e a} a^{4} W .
$$

The boundary conditions suggest that the appropriate solution for $W$ belonging to the lowest mode is

$$
W=A \sin (\pi z)
$$

where $A$ is a constant. Substituting Eq.(35) in Eq. (34) yields an expression for the electric Rayleigh number in the form

$$
R_{e a}=\frac{\delta^{8}\left(1+\Lambda_{c} \delta^{2}\right)}{a^{4}}-\frac{\delta^{2}}{a^{2}} R_{t}
$$

where $\delta^{2}=\pi^{2}+a^{2}$.

From Eq. (36) it is observed that $d R_{e a} / d \Lambda_{c}=\delta^{10} / a^{4}>0$ and hence increasing the value of couple stress parameter has a stabilizing effect on the system. But, it is noted that $d R_{e a} / d R_{t}=-\delta^{2} / a^{2}<0$ indicating the system gets destabilized with increasing value of $R_{t}$ (i.e., when the layer is heated from below), while opposite is the trend when the fluid layer is heated from above.

To find the critical value of $R_{e a}$, Eq. (36) is differentiated with respect to $a^{2}$ and equated to zero to get a polynomial in $\left(a_{c}^{2}\right)$ in the form

$$
\begin{aligned}
3 \Lambda_{c}\left(a_{c}^{2}\right)^{5}+2\left(1+5 \pi^{2} \Lambda_{c}\right)\left(a_{c}^{2}\right)^{4} & +2 \pi^{2}\left(2+5 \pi^{2} \Lambda_{c}\right)\left(a_{c}^{2}\right)^{3} \\
& -\pi^{2}\left(4 \pi^{4}+5 \pi^{6} \Lambda_{c}-R_{t}\right)\left(a_{c}^{2}\right)-2 \pi^{8}\left(1+\pi^{2} \Lambda_{c}\right)=0 .
\end{aligned}
$$


In the absence of couple stress (i.e., $L_{c}=0$ ), Eqs. (36) and (37) respectively reduce to

$$
\begin{aligned}
& R_{e a}=\frac{\delta^{2}}{a^{2}}\left(\frac{\delta^{6}}{a^{2}}-R_{t}\right) \\
& 2\left(a_{c}^{2}\right)^{4}+4 \pi^{2}\left(a_{c}^{2}\right)^{3}-\pi^{2}\left(4 \pi^{4}-R_{t}\right)\left(a_{c}^{2}\right)-2 \pi^{8}=0 .
\end{aligned}
$$

The above results coincide with those of Roberts (1969).

\subsection{Both boundaries rigid}

As in the case of free boundaries, an exact solution is not possible for the rigid boundaries and one has to resort to numerical methods to extract the critical stability parameters. For this, the Galerkin method is adopted to solve the resulting eigenvalue problem. To approximate the perturbed variables, the sets of spatial basis functions with constant coefficients are used:

$$
W=\sum A_{i} W_{i}, \Theta=\sum B_{i} \Theta_{i}, \Phi=\sum C_{i} \Phi_{i}
$$

where $A_{i}, B_{i}$ and $C_{i}$ are constants and the basis functions $W_{i}, \Theta_{i}$ and $\Phi_{i}$ will be represented by the power series satisfying the respective boundary conditions. Substituting Eq. (40) into Eqs. (31)-(33), multiplying the resulting momentum equation by $W_{j}(z)$, energy equation by $\Theta_{j}(z)$, electric potential equation by $\Phi_{j}(z)$; performing the integration by parts with respect to $\mathrm{z}$ between $\mathrm{z}=0$ and $\mathrm{z}=1$ and using the boundary conditions, leads to the following system of linear homogeneous algebraic equations:

$$
\begin{aligned}
& E_{j i} A_{i}+F_{j i} B_{i}+G_{j i} C_{i}=0 \\
& H_{j i} A_{i}+I_{j i} B_{i}=0 \\
& J_{j i} B_{i}+K_{j i} C_{i}=0
\end{aligned}
$$

where

$$
\begin{aligned}
& E_{j i}=<\Lambda_{c} D^{3} W_{j} D^{3} W_{i}+\left(1+3 a^{2} \Lambda_{c}\right) D^{2} W_{j} D^{2} W_{i}> \\
&+<a^{2}\left(2+3 a^{2} \Lambda_{c}\right) D W_{j} D W_{i}+a^{4}\left(1+a^{2} \Lambda_{c}\right) W_{j} W_{i}> \\
& F_{j i}=-\left(R_{t}+R_{e a}\right) a^{2}<W_{j} \Theta_{i}>, G_{j i}=R_{e a} a^{2}<W_{j} D \Phi_{i}>, H_{j i}=<\Theta_{j} W_{i}> \\
& I_{j i}=-<D \Theta_{j} D \Theta_{i}+a^{2} \Theta_{j} \Theta_{i}>, J_{j i}=-<\Phi_{j} D \Theta_{i}>, K_{j i}=-<D \Phi_{j} D \Phi_{i}+a^{2} \Phi_{j} \Phi_{i}>.
\end{aligned}
$$

Here the inner product is defined as $<f_{1} f_{2}>=\int_{0}^{1} f_{1} f_{2} d z$. 
The above set of homogeneous algebraic equations can have a non-trivial solution if and only if

$$
\left|\begin{array}{ccc}
E_{j i} & F_{j i} & G_{j i} \\
H_{j i} & I_{j i} & 0 \\
J_{j i} & 0 & K_{j i}
\end{array}\right|=0 .
$$

We select trial functions satisfying the appropriate boundary conditions as follows:

$$
W_{i}=\left(z^{2}-5 z^{4}+6 z^{5}-2 z^{6}\right) T_{i-1}^{*}, \Phi_{i}=\left(z-z^{2}\right) T_{i-1}^{*}, \Theta_{i}=\left(z-z^{2}\right) T_{i-1}^{*}
$$

where $T_{i}^{*}(i=1,2, \ldots . . n)$ is the modified Chebyshev polynomial of $i$ th order. Substituting the above trial functions in Eq. (39) and expanding the determinant leads to the characteristic equation giving the AC electric Rayleigh number $R_{e a}$ or the thermal Rayleigh number $R_{t}$ as a function of wave number $a$ as well as other parameters. The inner products involved in the determinant are evaluated analytically rather than numerically in order to avoid errors in the numerical integration. Numerical computations carried out reveal that the convergence in finding $R_{e a c}$ with respect to the wave number crucially depends on the value of $\Lambda_{c}$, and for higher values of $\Lambda_{c}$ more number of terms are found to be required in the Galerkin expansion. The results presented here are for $i=j=6$ the order at which the convergence is achieved, in general.

\section{Results and discussion}

The onset of convection in a layer of couple stress dielectric fluid heated uniformly either from below or from above is investigated under the influence of a uniform vertical AC electric field. The isothermal boundaries of the fluid layer are considered to be either free-free or rigid-rigid. It is shown that the principle of exchange of stability holds even in the presence of a vertical AC electric field. The eigenvalue problem is solved exactly in the case of free boundaries, while for rigid boundaries the eigenvalues are extracted numerically using the Galerkin technique. To validate the numerical procedure employed, first the test computations were carried out for rigid boundaries under the limiting case of $\Lambda_{c}=0$ (i.e., Newtonian case) and compared with the earlier published results. The critical AC electric Rayleigh number $R_{\text {eac }}$ and the corresponding wave number $a_{c}$ obtained for different values 
of $R_{t}$ are compared with those of Roberts (1969) in Table 1. The comparison shows excellent agreement of the numerical results and verifies the accuracy of the numerical method used.

Figures 2 and 3 respectively demonstrate the neutral curves in the $\left(R_{e a}, a\right)$ plane for various values of $\Lambda_{c}$ when $R_{t}=-500$ (heating from above) and for different values of $R_{t}$ when $\Lambda_{c}=0.1$. It is seen that the neutral curves exhibit single but different minimum with respect to the wave number for various values of physical parameters and also for two kinds of velocity boundary conditions. The region above each neutral curve corresponds to the unstable state of the system. The presence of couple stresses shows a significant effect on the stability characteristics of the system. It is observed that even small variations in the couple stress parameter amounts to significant change in the AC electric Rayleigh number. This is more so in the case of rigid boundaries (Fig. 2). As can be seen from Fig. 2, increasing $\Lambda_{c}$ is to increase the region of stability. Figure 3 illustrates that the region of stability increases for negative values of $R_{t}$ but the trend is reversed if the system is heated from below (i.e., $R_{t}>0$ ). From the figures it is also evident that, except for a quantitative change, the stability characteristics are identical for free-free and rigid-rigid boundaries.

To know the impact of physical parameters on the onset criterion, the critical AC electric Rayleigh number $R_{\text {eac }}$ is obtained with respect to the wave number $a$ for different values of physical parameters as well as for two types of velocity boundary conditions. The variations of $R_{\text {eac }}$ and $a_{c}$ as a function of $R_{t}$ are presented for different values of couple stress parameter $\Lambda_{c}$ in Figs. 4 (a) and (b), respectively. For any fixed value of $\Lambda_{c}$, increasing the value of thermal Rayleigh number amounts to decrease in the critical AC electric Raleigh number monotonically (Fig. 4a) indicating its effect is destabilizing. In other words, the presence of AC electric field is to augment heat transfer and to hasten the onset of convection in a layer of couple stress dielectric fluid. This is so irrespective of the velocity boundary conditions considered. This is due to the destabilizing electrical body force induced by the gradient of dielectric constant due to variations in temperature under the action of electric field which eventually drive an upward fluid motion. Nonetheless, increasing $\Lambda_{c}$ is to delay the onset of electrothermal convection due to the presence of couple stress. That is, the effect of suspended particles is to increase the viscosity of the fluid and thereby exhibits stabilizing influence on the system with increasing $\Lambda_{c}$. Moreover, the deviation in the critical values of 
$R_{\text {eac }}$ for different values of $\Lambda_{c}$ is found to be significant in the case of rigid boundaries when compared to free boundaries. From Fig. 4(a), it is also seen that the rigid-rigid boundaries are more stabilizing compared to free-free boundaries due to the increased suppression of disturbances in the case of rigid boundaries. Figure 4(b) shows that the critical wave number decreases with increasing $R_{t}$ and this trend is found to be insignificant in the case of rigidrigid boundaries. Furthermore, $a_{c}$ decreases with increasing $\Lambda_{c}$ for rigid boundaries case irrespective of the system heated from below or from above. However, a dual behavior is observed in the case of free boundaries. That is, the critical wave number decreases with increasing $\Lambda_{c}$ when the system is heated from above, but an opposite trend is noticed after $R_{t}(>0)$ exceeds certain value.

\section{Conclusions}

The criterion for the onset of convection in a couple stress dielectric fluid layer heated either from below or above subject to a vertical AC electric field has been investigated for free-free and rigid-rigid isothermal boundaries. The principle of exchange of stability is shown to be valid. The effect of increasing AC electric field is to enhance the heat transfer and to hasten the onset of convection, while the presence of couple stress inhibits the onset of electrothermal convection. Therefore, it appears the presence of couple stress enhances the performance of the microfluidic devices. It is observed that there is a qualitative agreement between the results of free-free and rigid-rigid boundaries. However, the presence of couple stresses exhibit significant effect on the stability of the system for the case with rigid boundaries compared to the free boundaries case. The foregoing results give an insight for the coupling effect of electrical body force, buoyancy force and the couple stresses on the stability characteristics of a dielectric fluid layer for two types of velocity boundary conditions.

\section{Acknowledgements}

The second author (MA) thanks the UGC, New Delhi for granting fellowship under Special Assistance Program CAS Phase-II. The third author (CON) was supported by the Research Grants Council of the Hong Kong Special Administrative Region, China, under Project HKU $715510 \mathrm{E}$. 


\section{References}

Bradley, R. (1978). Overstable electroconvective instabilities. Quarterly Journal of Mechanics and Applied Mathematics, 31, 381-390.

Chang, M. H., Ruo, A. C., \& Chen, F. (2009). Electrohydrodynamic instability in a horizontal fluid layer with electrical conductivity gradient subject to a weak shear flow. Journal of Fluid Mechanics, 634, 191-215.

Eringen, A. C. (1966). Theory of micropolar fluids. Journal of Mathematics and Mechanics, $16,1-18$.

Jones, T. B. (1978). In T. F. Irvine, Jr. \& J. P. Hartnett (Eds.). Electrohydrodynamically enhanced heat transfer in liquids-A review in Advances in Heat Transfer. Academic Press, 107-144.

Lin, H. (2009). Electrokinetic instability in microchannel flows: A review. Mechanical Research Communications, 36, 33-38.

Maekawa, T., Abe, K., \& Tanasawa, I. (1992). Onset of natural convection under an electric field. International Journal of Heat and Mass Transfer, 35, 613-621.

Malashetty, M. S., Gaikwad, S. N., \& Swamy, M. (2006). An analytical study of linear and non-linear double diffusive convection with Soret effect in couple stress liquids. International Journal of Thermal Science, 45, 897-907.

Othman, M. I. A. (2001). Electrohydrodynamic stability in a horizontal viscoelastic fluid layer in the presence of a vertical temperature gradient. International Journal of Engineering Science, 39, 1217.

Othman, M. I. A., \& Sweilam, N. H. (2002). Electrohydrodynamic instability in a horizontal viscoelastic fluid layer in the presence of internal heat generation. Canadian Journal of Physics, 80, 697-705.

Othman, M. I. A., \& Zaki, S. A. (2003). The effect of thermal relaxation time on a electrohydrodynamic viscoelastic fluid layer heated from below. Canadian Journal of Physics, 81, 779-787.

Othman, M. I. A. (2004). Electrohydrodynamic instability of a rotating layer of a viscoelastic fluid heated from below. Journal of Applied Mathematics and Physics (ZAMP), 55, 468482.

Pontiga, F., \& Castellanos, A. (1994). Physical mechanisms of instability in a liquid layer subjected to an electric field and a thermal gradient. Physics of Fluids, 6, 1684-1701.

Roberts, P. H. (1969). Electrohydrodynamic convection. Quarterly Journal of Mechanics and Applied Mathematics, 22, 211-220. 
Ruo, A. C., Chang, M. H., \& Chen, F. (2010). Effect of rotation on the electrohydrodynamic instability of a fluid layer with an electrical conductivity gradient, Physics of Fluids, 22, 024102.

Saville, D. A. (1997). Electrohydrodynamics: The Taylor-Melcher leaky dielectric model. Annual Review of Fluid Mechanics, 29, 27-64.

Sharma, R. C., \& Sharma, M. (2004). Effect of suspend particle and couple stress fluid heated from below in the presence of rotation and magnetic field. Indian Journal of Pure and Applied Mathematics, 35, 973-989.

Shivakumara, I. S., Nagashree, M. S., \& Hemalatha, K. (2007). Electroconvective instability in a heat generating dielectric fluid layer. International Communications in Heat and Mass Transfer, 34, 1041-1047.

Shivakumara, I. S., Rudraiah, N., \& Hemalatha, K.(2009). Electrothermoconvection in a dielectric fluid layer in the presence of heat generation. International Journal of Applied Mathematics, 1, 87-101.

Shivakumara, I. S., Lee, J., Vejravelu, K., \& Akkanagamma, M. (2012). Electrothermal convection in a rotating dielectric fluid layer: Effect of velocity and temperature boundary conditions. International Journal of Heat and Mass Transfer, 55, 2984-2991.

Smorodin, B. L. (2001). The onset of convection of a poorly conducting fluid in a modulated thermal field. Journal of Experimental and Theoretical Physics, 93, 1231-1238.

Stokes, V. K. (1966). Couple stresses in fluids. Physics of Fluids, 91, 1709-1715.

Straughan, B. (2004). The energy method, stability, and nonlinear convection (second edition). Springer.

Sunil, Devi, R., \& Mahajan, A. (2011). Global stability for thermal convection in a couplestress fluid. International Communications in Heat and Mass Transfer, 55, 2984-2991.

Takashima, M., \& Aldridge, K. D. (1976). The stability of a horizontal layer of dielectric fluid under the simultaneous action of a vertical d.c. electric field and vertical temperature gradient. Quarterly Journal of Mechanics and Applied Mathematics, 29, 71-87.

Takashima, M., \& Ghosh, A. K. (1979). Electrohydrodynamic instability in a viscoelastic fluid layer. Journal of Physical Society of Japan, 47, 1717-1722.

Turnbull, R. J. (1968). Electroconvective instability with a stabilizing temperature gradient, I and II: Theory and experimental results. Physics of Fluids, 11, 2588-2603. 


\begin{tabular}{|c|c|c|c|c|}
\hline \multicolumn{3}{|c|}{ Roberts (1969) } & \multicolumn{2}{c|}{ Present study } \\
\hline$R_{t}$ & $R_{\text {eac }}$ & $a_{c}$ & $R_{\text {eac }}$ & $a_{c}$ \\
\hline-1000 & 3370.077 & 3.2945 & 3374.4899 & 3.2924 \\
\hline-500 & 2749.868 & 3.2598 & 2754.1106 & 3.2581 \\
\hline 0 & 2128.696 & 3.2260 & 2132.7933 & 3.2245 \\
\hline 500 & 1506.573 & 3.1929 & 1510.5526 & 3.1917 \\
\hline 1000 & 883.517 & 3.1606 & 887.4040 & 3.1597 \\
\hline 1707.762 & 0.0 & 3.1162 & 0.0 & 3.1158 \\
\hline
\end{tabular}

Table 1: Comparison of $R_{\text {eac }}$ and $a_{c}$ for different values of $R_{t}$ for rigid-rigid boundaries when $\Lambda_{c}=0$.

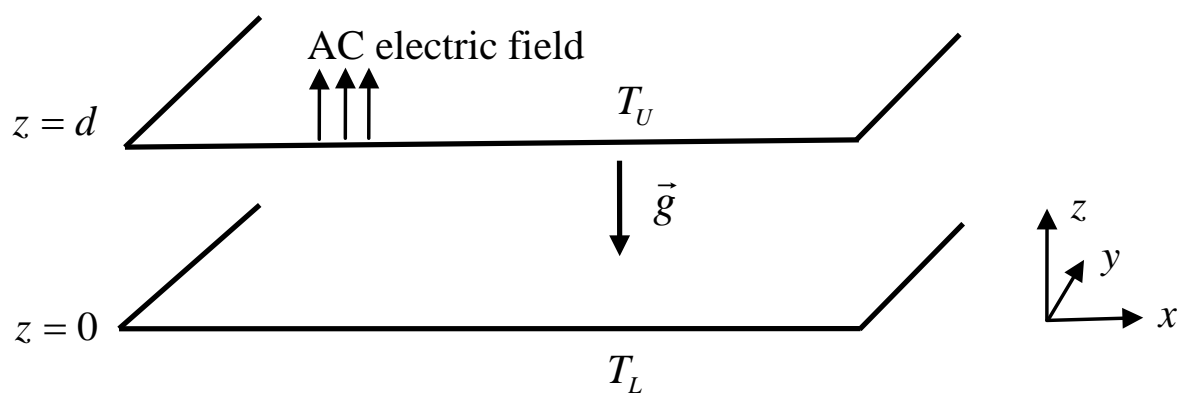

Fig. 1. Physical Configuration 


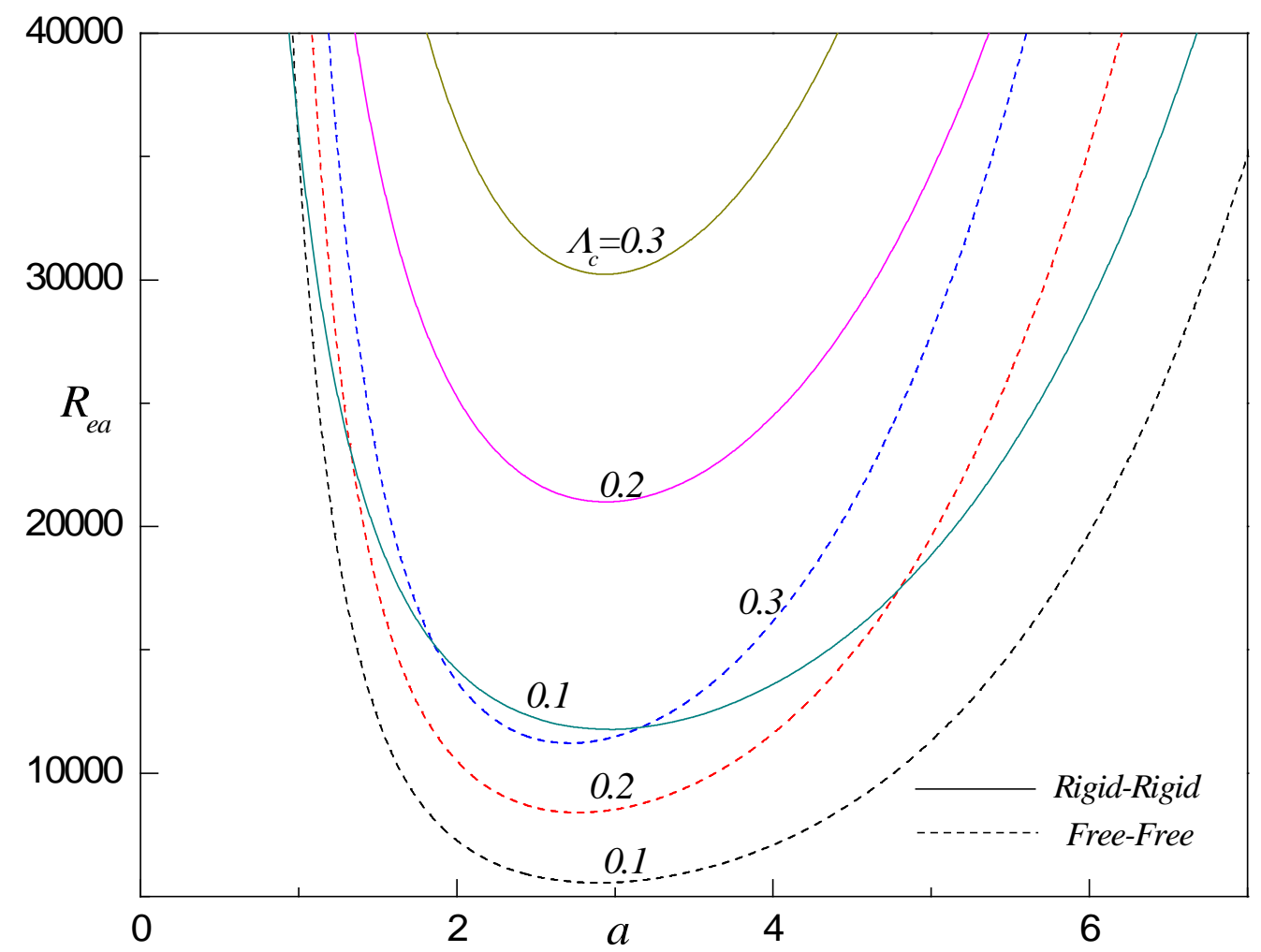

Fig. 2. Neutral curves for different values of $\Lambda_{c}$ with $R_{t}=-500$

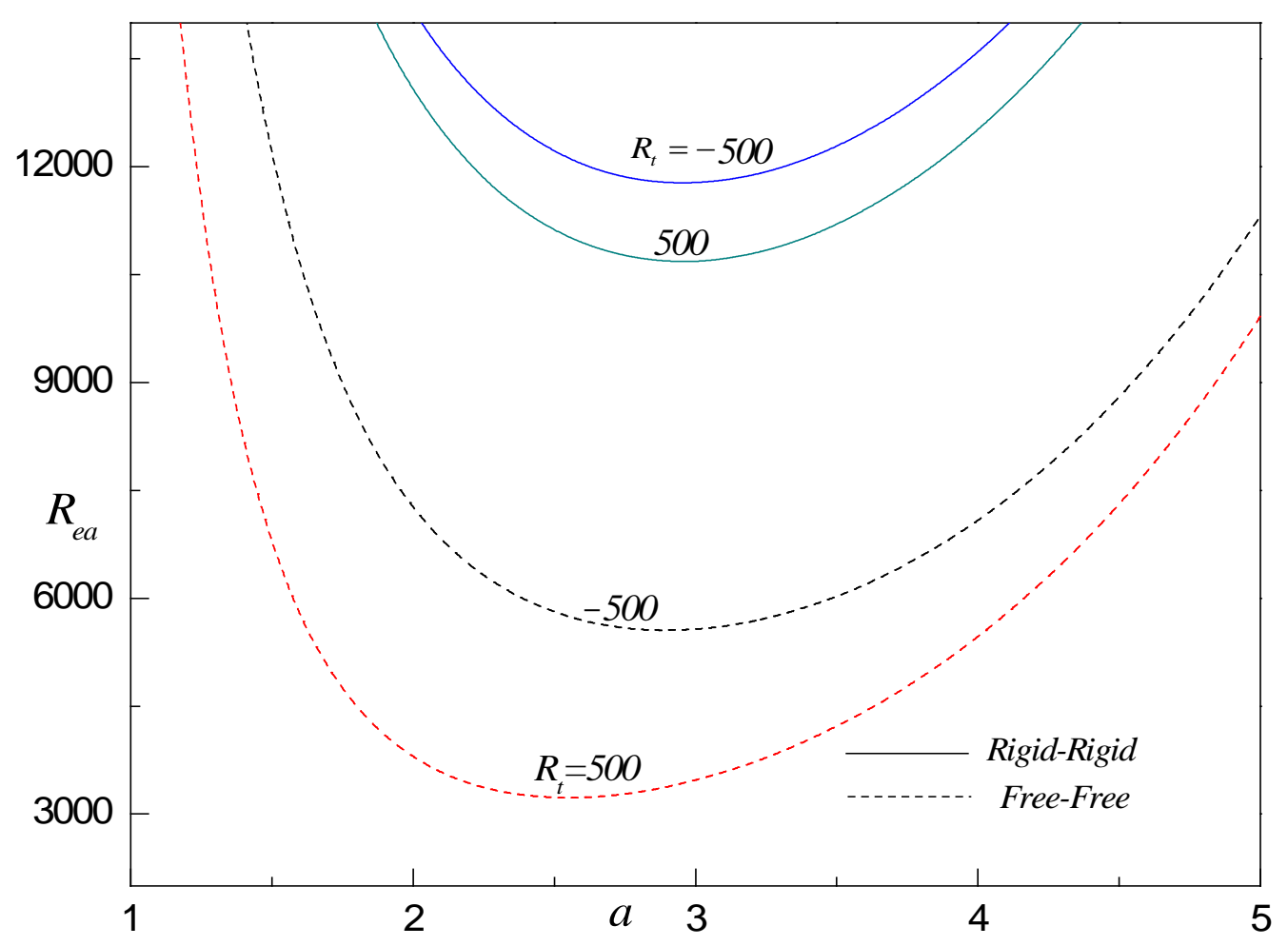

Fig. 3. Neutral curves for two values of $R_{t}$ with $\Lambda_{c}=0.1$. 

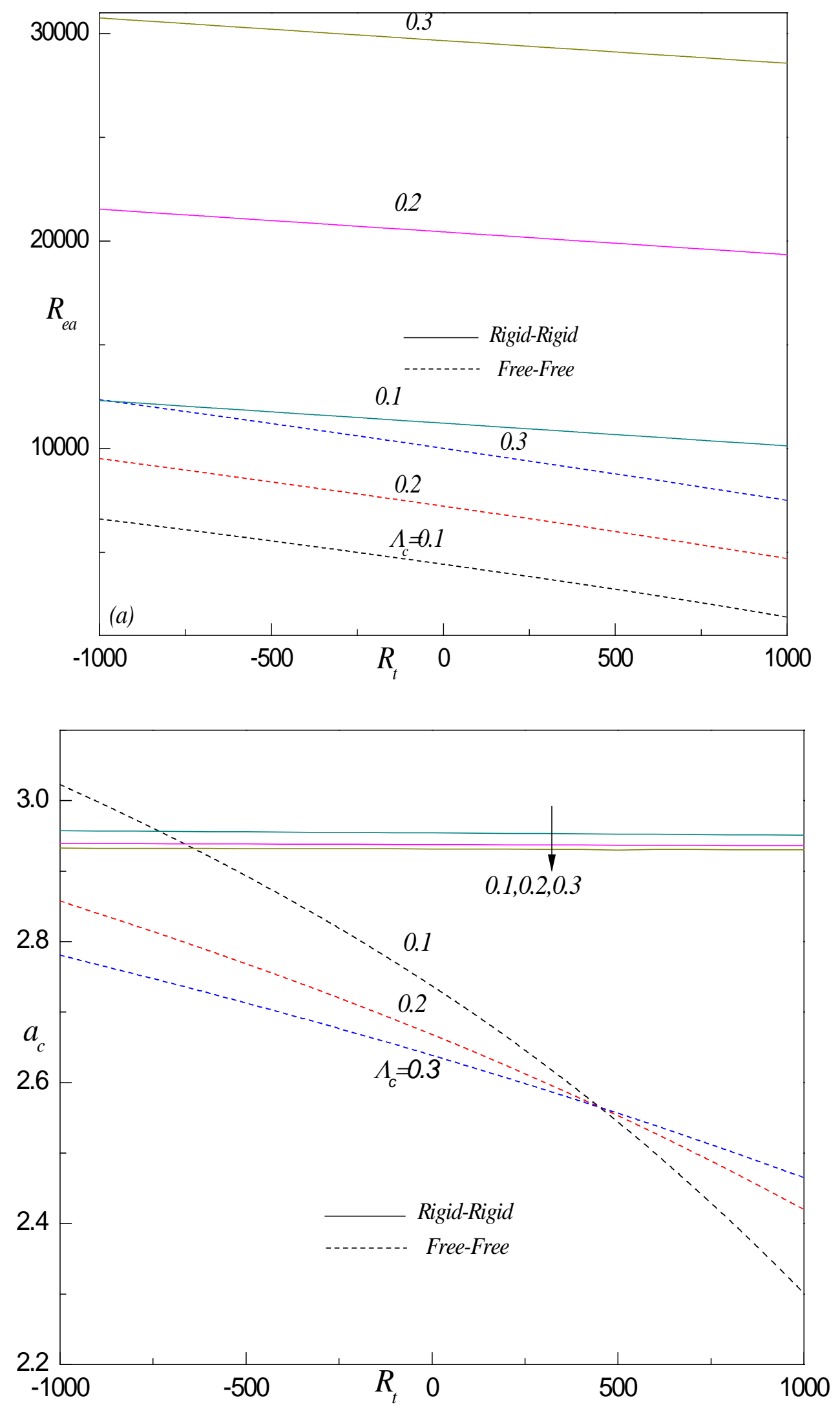

Fig. 4. Variation of (a) $R_{\text {eac }}$ and (b) $a_{c}$ as a function of $R_{t}$ for different values of $\Lambda_{c}$ 